\title{
Conocimientos, conductas y motivos en los métodos preventivos para prácticas sexuales ororeceptivas en estudiantes de odontología
}

\section{Knowledge, behaviors and motives in preventive methods for ororeceptive sexual practices in dentistry students.}

\author{
Godoy-Flores Francisca ${ }^{1}$, Lee-Muñoz Ximena ${ }^{1^{*}}$
}

1. Universidad de Chile, CEVEO, Facultad de Odontología - Santiago, Chile.

* Correspondencia autor. Ximena Lee Muñoz Sergio Livingstone \#943. Comuna Independencia. Región Metropolitana. | Teléfono: +56999914996 | E-mail:xlee@odontologia.uchile.cl Trabajo recibido el 09/10/2018.

Aprobado para su publicación el 27/11/2018

\section{RESUMEN}

La odontología está en búsqueda de miradas más integradoras, una de ellas se relaciona con la sexualidad. La educación sexual en odontología, ha tenido escasa representación curricular, instalándose más desde lo curativo que de lo preventivo. Este estudio evaluó el grado de conocimiento, las conductas y motivos relativos a los métodos preventivos y al riesgo asociado a las prácticas de sexo oral, en estudiantes de odontología de una universidad chilena. Material y métodos: Este estudio cuantitativo, transversal y analítico, utilizó como instrumento de recogida de datos, una encuesta anónima y autoaplicada, en 50 estudiantes voluntarios. Este instrumento indagó acerca de las condiciones relativas a salud sexual, específicamente en prácticas de sexo oral. Los análisis estadísticos fueron realizados utilizando los programas SPSS $®$ v20 y Excel $($. Resultados: En cuanto a los conocimientos de métodos preventivos para prácticas de sexo oral, además del condón, otras barreras fueron poco conocidas (13\%). El $90 \%$ reportó haber practicado sexo oral alguna vez, de estos, $78 \%$ no utilizó barreras de protección. De los que reportaron haber usado barrera, el 100\% utilizó condón. Conclusiones: Los conocimientos, conductas y motivos en salud sexual denotan un conocimiento deficiente relativo a métodos preventivos para prácticas de sexo oral y su riesgo asociado.

PALABRAS CLAVE:

Sexo oral; Prácticas sexuales.

Rev. Clin. Periodoncia Implantol. Rehabil. Oral Vol. 12(1); 23-26, 2019.

\section{ABSTRACT}

Dentistry is in search of more inclusive points of view, one of them related to sexuality. Sexual education has had little curricular representation in Dentistry, being approached more from a curative point of view rather than a preventive one. This study evaluated the degree of knowledge, conduct and motives related to preventive methods and the risk associated with oral sex practices among dental students of a Chilean university. Materials and methods: This quantitative, transversal and analytical study used an anonymous and self-applied survey on 50 voluntary students. The instrument inquired about conditions related to sexual health, specifically oral sex practices. Statistical data was analyzed using softwares such as SPSS v20 and Excel. Results: In terms of knowledge about preventive methods for oral sex practices, aside from condoms, other barriers where little known (13\%); $90 \%$ reported having practiced oral sex once, and among these, $78 \%$ didn't use protection barriers. Of those who reported having used a barrier, $100 \%$ used condoms. Conclusions: Knowledge, conducts and motives in sexual health report a deficient knowledge of preventive methods for oral sex practices and their associated risk.

KEY WORDS:

Oral sex; Sexual activities.

Rev. Clin. Periodoncia Implantol. Rehabil. Oral Vol. 12(1); 23-26, 2019. 


\section{INTRODUCCIÓN}

La sexualidad constituye un comportamiento que abarca diferentes aspectos como el sexo, género y reproducción, entre otros ${ }^{(1,2)}$. Uno de los ámbitos relativos a sexualidad es la salud sexual, que incluye el acceso a una educación sexual integral, con políticas públicas destinadas a la protección y prevención ${ }^{(3,4,5)}$

La actividad sexual es una expresión conductual evidenciada a través de prácticas sexuales (PS) coitales o no coitales. Una práctica de tipo no coital es el sexo oral (SO), donde la boca actúa como un órgano emisor de estímulos y de percepción sensorial|(6,7,8) . Los distintos tipos de SO son cunnilingus, fellatio y annilingus ${ }^{(9)}$. Según el desarrollo psicosexual la boca se relaciona con las pulsiones sexuales ${ }^{(10,11)}$, pues posee una libido instintiva que se desarrolla en etapas ${ }^{(12)}$. De esta forma, la boca está cargada libidinalmente, movilizando la pulsión del sujeto, donde por ejemplo, la succión se configura como una actividad erótica ${ }^{(13)}$

El SO se considera de riesgo, debido a la exposición a infecciones de transmisión sexual (ITS) orales y/o genitales provenientes de sus fluidos. Mientras mayor sea el contacto con fluidos, mayor es el riesgo de infección de tipo alto, moderado o mínimo (Tabla 1) ${ }^{(14,15)}$. Las ITS constituyen un grupo de patologías cuyos mecanismos de transmisión son de tipo sexual o no sexual: Entre los patógenos que se transmiten por contacto sexual, varios se vinculan a la incidencia de ITS como VIH y virus del papiloma humano (VPH), entre otras. De las no sexuales, la clamidiasis, gonorrea, VIH y sífilis pueden transmitirse durante el embarazo o el parto. En Chile, el MINSAL ha elaborado normas de manejo, estableciendo disposiciones y lineamientos para su atención y educación, debido a que la OMS ha determinado que un millón de personas adquieren una ITS cada día en el mundo ${ }^{(16)}$.

Respecto de la percepción de riesgo, existe tendencia a minimizar los

Tabla 1. Categorización de la valoración del riesgo ante una exposición sexual (OPS, 2013).

\begin{tabular}{|c|c|c|}
\hline Riesgo Alto & Riesgo moderado & Riesgo mínimo \\
\hline $\begin{array}{l}\text { - Recepción: } \\
\text { - Anal con } \\
\text { eyaculación, sin } \\
\text { preservativo o con } \\
\text { preservativo que ha } \\
\text { sufrido rotura o ha } \\
\text { sido mal utilizado. } \\
\text { - Vaginal con } \\
\text { eyaculación, sin } \\
\text { preservativo, con } \\
\text { rotura o mal uso. } \\
\text { - Anal sin } \\
\text { eyaculación. }\end{array}$ & $\begin{array}{l}\text { - Recepción vaginal } \\
\text { sin eyaculación. } \\
\text { - Penetración } \\
\text { vaginal sin } \\
\text { preservativo con } \\
\text { rotura o mal uso. } \\
\text { - Sexo oro-genital } \\
\text { receptivo o activo } \\
\text { con eyaculación, } \\
\text { sin preservativo, } \\
\text { o con rotura o mal } \\
\text { uso. }\end{array}$ & $\begin{array}{l}\text { - SO sin } \\
\text { eyaculación. } \\
\text { - Recepción } \\
\text { vaginal con } \\
\text { preservativo. } \\
\text { - Penetración } \\
\text { vaginal o rectal } \\
\text { con preservativo } \\
\text { utilizado } \\
\text { correctamente. }\end{array}$ \\
\hline
\end{tabular}

efectos derivados de estas prácticas, por lo que la prevalencia de las PS no coitales ha aumentado ${ }^{(17)(18)}$. En Chile, del $71 \%$ de los jóvenes entre 15 y 29 años iniciados sexualmente, $49 \%$ ha practicado SO cuya frecuencia aumenta con la edad y es más reportada en hombres (52\%) que mujeres $(46 \%)$. Quienes han practicado SO, $97 \%$ ha mantenido PS coitales, evidenciando que ambas conductas están relacionadas. Otros estudios indican que entre un $14 \%$ y $50 \%$ han tenido SO antes de su primera experiencia coital, develando que se trataría de un comportamiento que acompaña al inicio sexual ${ }^{(19,20)}$. Es importante destacar que en su práctica pocos utilizan barreras de protección (BP) para ITS ${ }^{(21)}$.

Aunque el riesgo de transmisión de ITS es mayor en las PS vaginal/ anal que en el $\mathrm{SO}$, el aumento de la práctica de este último y la escasa utilización de BP, relevan al SO como mecanismo de transmisión de ITS ${ }^{(22)}$. Dentro de las BP que reducen el riesgo de adquirir o transmitir una ITS, se encuentra el condón, gomas diques y máscaras, entre otras. Además, se debe evitar la exposición a fluidos especialmente cuando existan lesiones orales o genitales. Un estudio indagó dichas prácticas y utilización de BP en adolescentes, desvelando que $9 \%$ experimentó fellatio con eyaculación y $10 \%$ cunnilingus. El $86 \%$ no usó condón y $8 \%$ sí y el uso de la goma dique durante el cunnilingus fue menor en comparación a otras barreras ${ }^{(23)}$.

Por tener un carácter de compromiso con la salud pública, se han generado normas de manejo y tratamiento de las ITS. Esta iniciativa nace desde lo médico dejando fuera lo odontológico, lo que genera un vacío cognitivo y una deuda social, por lo que el propósito de este estudio es evaluar el grado de conocimiento, conductas y motivos en torno a los métodos preventivos y al riesgo asociado a las prácticas de $\mathrm{SO}$, en estudiantes de odontología.

\section{MATERIALES Y MÉTODOS}

En este estudio cuantitativo de tipo observacional y transversal, participaron 87 estudiantes de odontología (EO) que cursaban el quinto año de la carrera seleccionados a través de un muestreo no probabilístico intencionado (Tabla 2). De este número de estudiantes, 50 cumplieron con los criterios de inclusión y aceptaron participar los cuales firmaron el consentimiento informado, aprobado por el Comité Ético Científico de la Facultad en estudio. La justificación de trabajar con este grupo es que se esperaría que contaran con cierto dominio temático en prevención de ITS, independiente de si ha iniciado su vida sexual. La universidad participante se mantiene en anonimato por consideraciones éticas.

Se utilizó una encuesta de tipo estructurada, anónima y autoadministrada, basada en la Octava Encuesta Nacional de Juventud de 2015 (disponible en

Tabla 2. Criterios de inclusión y exclusión del estudio

\begin{tabular}{|l|l|}
\hline \multicolumn{1}{|c|}{ Criterios de inclusión } & \multicolumn{1}{|c|}{ Criterios de exclusión } \\
\hline - Estudiantes de la carrera de & • Estudiantes mayores a \\
odontología de una universidad & $\begin{array}{l}\text { 29 años, que no acepten } \\
\text { participar en el estudio, de } \\
\text { chilena. }\end{array}$ \\
$\begin{array}{l}\text { - Rango etario: } 18-29 \text { años. } \\
\text { cursos inferiores a quinto año } \\
\text { de la carrera.y aquellos que } \\
\text { participaron del pilotaje de la } \\
\text { del año 2017. }\end{array}$ & encuesta. \\
- Aceptar y firmar el \\
consentimiento informado.
\end{tabular}

http://www.injuv.gob.cl), a la cual se le hicieron algunas modificaciones para este estudio, autorizadas por INJUV. Se incluyeron ítems de caracterización general, conocimientos de métodos preventivos y de ITS. El carácter autoadministrado se debió a que existen temas que son sensibles en tres dimensiones: deseabilidad social, privacidad y confidencialidad en cuanto a su propia práctica sexual.

Previo a la aplicación del instrumento se procedió a hacer aportes de validez de contenido de la encuesta mediante un juicio de expertos (constituido por una odontóloga, un enfermero y una matrona) que fueron seleccionados por su experiencia en el trabajo con jóvenes, contar con algún curso en sexualidad y por su disponibilidad y motivación para participar. Éstos, previa firma de un consentimiento informado, respondieron una pauta de evaluación por jueces del instrumento en torno a la pertinencia de los ítems respecto a la dimensión para la que estaban formulados, al propósito del estudio y claridad de formulación. Posterior a la evaluación por expertos y a la incorporación de cambios sugeridos por éstos, se procedió a la realización de una prueba piloto en cinco estudiantes con las mismas características de la población a estudiar y que aceptaron voluntariamente participar. El objetivo de este pilotaje fue evaluar la claridad del instrumento, el tiempo de respuesta y estandarizar la forma de aplicación. En esta prueba piloto se les solicitó responder poniendo énfasis en la claridad de formulación de los ítems y el contenido de éstos. A su vez, si tenían una observación o sugerencia, podían anotarla en el espacio del ítem respectivo. Una vez sistematizadas las sugerencias de los estudiantes en la prueba piloto, se modificaron aquellos ítems que presentaron dificultades, obteniendo el instrumento en su versión final para la aplicación a la población objetivo.

La aplicación de la encuesta final se realizó en un lugar y tiempo específicos; las respuestas se recibieron individualmente en sobres cerrados. Para el plan de análisis de datos se utilizaron los programas SPSS Stata $\mathrm{v}^{2} \mathrm{O}^{\circledR}$ y Excel $^{\circledR}$. Para variables categóricas se utilizaron frecuencias absolutas y porcentajes. Para las cuantitativas, se utilizaron medidas de tendencia central y de dispersión (desviación estándar=DE). Para la comparación de grupos en función de variables categóricas, se utilizaron tablas de contingencia y chi-cuadrado. Para la comparación de grupos en función de variables cuantitativas, se utilizó $t$ de Student. Se consideró un nivel de significación del $5 \%$.

\section{RESULTADOS}

De los 50 voluntarios, $78 \%(n=38)$ fueron mujeres y $24 \%(n=12)$ hombres.

En la Tabla 3, se describe el grado de conocimiento acerca de BP según PS. De las conductas asociadas a SO, $90 \%(n=45)$ reportó haberlas practicado y $10 \%(n=5)$ no, con un promedio de 1,42 parejas $(D E=1,53)$ y una mediana de 1 pareja en el último año. El que la media sea más alta se debió a casos que reportaron mayor cantidad de parejas. De aquellos 
Tabla 3. Conocimiento de barreras mecánicas de protección para prácticas sexuales

\begin{tabular}{|c|c|c|}
\hline Barreras mecánicas & $\mathbf{N}^{\circ}$ & $\begin{array}{l}\text { Porcentaje de casos que } \\
\text { reportan conocerlo \% }\end{array}$ \\
\hline Condón masculino & 48 & 98 \\
\hline Condón femenino & 33 & 67,3 \\
\hline Máscara para SO & 5 & 10,2 \\
\hline Lámina para SO & 6 & 12,2 \\
\hline Goma dique para SO & 5 & 10,2 \\
\hline Dedo de látex & 5 & 10,2 \\
\hline Ninguno & 1 & 2 \\
\hline
\end{tabular}

que practicaron SO, $78 \%(n=35)$ no utilizó protección y $22 \%(n=10)$, sí. De aquellos que utilizaron barrera, el 100\% indicó condón. De las prácticas de SO según género, $89,5 \%(n=34)$ de las mujeres practicaron SO y $10,5 \%(n=4)$, no. En hombres, un $91,7 \%(n=11)$ practicó SO y $8,3 \%(n=1)$, no. No se presentó asociación significativa entre género y prácticas de SO (chi-cuadrado de Pearson=0,049; $p=0,825$ ). Al asociar este mismo ítem según orientación sexual, de los heterosexuales un $19,5 \%(n=8)$ utilizó BP y $80,5 \%(n=33)$, no. De los participantes homosexuales, $66,7 \%(n=2)$ utilizó BP durante SO y 33,3\%(n=1), no. No hubo asociación significativa entre orientación sexual y uso de protección para SO debido al número de participantes (chi-cuadrado de Pearson=3,88; $p=0,14$ ).

Para el número de parejas según género para prácticas de $\mathrm{SO}$, en mujeres fue 1,11 ( $D E=0,72)$ y en hombres 2,36 ( $D E=2,69)$. Para ambos géneros la mediana de parejas para SO fue 1 . No hubo diferencia significativa en la cantidad de parejas para esta práctica según género ( $\mathrm{t}=-$ $1,516 ; p=0,159)$.

Respecto del VIH y VPH, las Tablas 4 y 5 , muestran conductas riesgosas y no riesgosas en la transmisión de estos virus respectivamente. Finalmente, respecto a ITS, el $4 \%(n=2)$ reportó haber presentado una alguna vez, siendo en ambos casos VPH.

\section{DISCUSIÓN}

La odontología está en constante búsqueda de miradas más integradoras, y en el caso específico de lo que se expone de esta investigación, una de ellas se relaciona con la sexualidad humana. Sin embargo, la educación sexual en odontología ha tenido escasa representación curricular, instalándose más desde lo curativo que de lo preventivo, lo cual ha quedado develado en los resultados obtenidos en este estudio.

En cuanto a las barreras de protección para prácticas de sexo oral entre estudiantes de odontología, se evidencia que además del condón, éstas son poco conocidas ( $13 \%$ de respuestas). Cabe destacar que una de las barreras de protección mencionadas fue la goma dique, material de uso odontológico del cual se desconoce que puede ser indicado como tal. Otros estudios de prácticas y conocimientos en salud sexual en estudiantes de odontología, refieren que el condón es considerado como un método anticonceptivo y preventivo de infecciones de transmisión sexual (ITS), pero no se relaciona con sexo oral ${ }^{(24)}$.

En este estudio, el $90 \%$ de la muestra declaró practicar sexo oral, en comparación con un $49 \%$ de la encuesta del Instituto Nacional de la Juventud (INJUV). Esto puede deberse a que INJUV incluyó a jóvenes de 15 a 29 años y al desagregar los datos por variables de segmentación, se obtiene que su frecuencia aumenta con la edad (63,7\% entre los $25-29$ años). De aquellos que declararon no utilizar protección, los datos son similares a los obtenidos en una investigación en estudiantes de odontología colombianos, donde el 80,5\% tampoco utilizó barrera de protección ${ }^{(24)}$. Esto evidencia una falta de conocimiento del riesgo de contraer ITS por esta vía. Aquellos que reportaron haber utilizado alguna barrera de protección, indicaron solo al condón, lo que indica que conocen y utilizan pocas barreras para sexo oral.

De las prácticas de sexo oral según género, las mujeres lo reportaron en un $89,5 \%$ y los hombres en $91,7 \%$. En la encuesta INJUV, estas fueron reportadas por $52 \%$ de hombres y $46 \%$ de mujeres. La media de parejas fue mayor en hombres que mujeres, pero esta diferencia se debió a la presencia de casos atípicos en hombres de la muestra.

En cuanto al nivel de conocimiento respecto del contagio con VIH, este es mayor entre estudiantes de odontología $(E O)$ en comparación con los
Tabla 4. Conocimiento relativo a conductas riesgosas y no riesgosas de transmisión del VIH

\begin{tabular}{|c|c|c|c|c|}
\hline & & $\begin{array}{l}\text { Sí } \\
\%\end{array}$ & $\begin{array}{c}\text { No } \\
\%\end{array}$ & $\begin{array}{c}\text { No sé } \\
\%\end{array}$ \\
\hline Riesgosas & $\begin{array}{l}\text { Compartiendo objetos } \\
\text { cortantes con personas } \\
\text { que viven con VIH/SIDA }\end{array}$ & 78 & 10 & 12 \\
\hline \multirow{2}{*}{$\begin{array}{l}\text { (Respuesta } \\
\text { correcta= Sí, } \\
\text { si se puede } \\
\text { transmitir) }\end{array}$} & $\begin{array}{c}\text { A través de una } \\
\text { transfusión de sangre } \\
\text { infectada con VIH/SIDA }\end{array}$ & 100 & 0 & 0 \\
\hline & $\begin{array}{l}\text { Teniendo relaciones } \\
\text { sexuales sin usar } \\
\text { condón con personas } \\
\text { que viven con VIH/SIDA }\end{array}$ & 100 & 0 & 0 \\
\hline No riesgosas & $\begin{array}{c}\text { Compartiendo comida } \\
\text { con personas que viven } \\
\text { con VIH/SIDA }\end{array}$ & 2 & 94 & 4 \\
\hline \multirow{2}{*}{$\begin{array}{c}\text { (Respuesta } \\
\text { correcta= } \\
\text { No, no } \\
\text { se puede } \\
\text { transmitir) }\end{array}$} & $\begin{array}{l}\text { Bañándose en piscinas } \\
\text { públicas con personas } \\
\text { que viven con VIH/SIDA }\end{array}$ & 0 & 94 & 6 \\
\hline & $\begin{array}{l}\text { Compartiendo baños } \\
\text { públicos con personas } \\
\text { portadoras de VIH }\end{array}$ & 0 & 94 & 6 \\
\hline
\end{tabular}

Tabla 5. Conocimiento relativo a conductas riesgosas y no riesgosas de transmisión del VPH

\begin{tabular}{|c|c|c|c|c|}
\hline & & $\begin{array}{l}\text { Sí } \\
\%\end{array}$ & $\begin{array}{l}\text { No } \\
\%\end{array}$ & $\begin{array}{c}\text { No sé } \\
\%\end{array}$ \\
\hline Riesgosas & $\begin{array}{l}\text { Teniendo relaciones } \\
\text { sexuales con } \\
\text { penetración vaginal sin } \\
\text { usar protección, con } \\
\text { personas portadoras } \\
\text { de VPH }\end{array}$ & 98 & 2 & 0 \\
\hline $\begin{array}{l}\text { (Respuesta } \\
\text { correcta= Sí, } \\
\text { si se puede } \\
\text { transmitir) }\end{array}$ & $\begin{array}{l}\text { Teniendo SO sin } \\
\text { protección con una } \\
\text { persona portadora del } \\
\text { VPH }\end{array}$ & 95,9 & 2 & 2 \\
\hline No riesgosas & $\begin{array}{l}\text { Tener un antecedente } \\
\text { familiar hereditario de la } \\
\text { enfermedad (VPH) }\end{array}$ & 20,4 & 30,6 & 49 \\
\hline $\begin{array}{l}\text { (Respuesta } \\
\text { correcta= } \\
\text { No, no } \\
\text { se puede } \\
\text { transmitir) }\end{array}$ & $\begin{array}{l}\text { Carencia de hábitos de } \\
\text { higiene }\end{array}$ & 15,9 & 52,3 & 31,8 \\
\hline
\end{tabular}

de INJUV ( $81 \%$ y $80 \%$, respectivamente). Llama la atención que en ambos estudios pocos reconocen como conducta de riesgo el "compartir objetos cortantes con personas que viven con $\mathrm{VIH}$ " (78\% y $52 \%$ respectivamente). Las formas no riesgosas de transmisión fueron reconocidas correctamente en menor proporción que aquellas sí riesgosas, indicando la existencia de mitos respecto a la adquisición del VIH, que pueden ser base de discriminación hacia quienes viven con esta condición.

Respecto de la realización del test de Elisa o de VIH, en la encuesta INJUV y entre estudiantes de odontología fue de $30 \%$ y $44 \%$ respectivamente. Si bien el porcentaje es mayor en estos, se considera bajo para el total iniciado sexualmente. Algunas razones por las cuales los estudiantes de odontología se realizaron el test fue por exposición a una situación de riesgo, incluido el accidente cortopunzante. De aquellos que no se han realizado el test, la respuesta más frecuente fue el mantener pareja estable, especialmente entre estudiantes de odontología. En la encuesta INJUV, el 
porcentaje atribuido a la misma razón disminuye notoriamente develando que, con la edad, aumenta el número de parejas estables: $9 \%$ entre los 15 a 19 años a $34 \%$ entre los 25 a 29 años.

Respecto del test de VIH, más de la mitad requiere información respecto a cómo solicitar el test o el lugar donde se realiza, lo cual repercute en el quehacer profesional al momento de informar a un paciente. Respecto del virus papiloma humano (VPH), las conductas riesgosas fueron reconocidas por casi la totalidad de la muestra, pero las no riesgosas lo fueron en menor proporción. El tener un antecedente familiar hereditario de VPH puede haber generado confusión por su relación con cáncer del cuello uterino o por la posibilidad de transmisión vertical; la evidencia demuestra que el riesgo de transmisión vertical a las mucosas oral o genital de recién nacidos es variable ${ }^{(25)}$. Sin embargo, en ninguno de los casos anteriores la transmisión del VPH estaría actuando como una enfermedad hereditaria. Los estudiantes de odontología presentan deficiencias para reconocer correctamente las formas no efectivas de transmisión denotando falta de conocimientos respecto del contagio.

En cuanto a la prevalencia de ITS, un bajo porcentaje reportó haber presentado una, siendo en aquellos casos VPH. EI VPH es la ITS más frecuente a nivel mundial(26). Si bien el riesgo de contraerla a través del sexo oral es menor, las investigaciones han indicado que la transmisión por vía oral es una preocupación, especialmente porque se considera erróneamente al sexo oral como una práctica no riesgosa. Existe escasa bibliografía con respecto a esta y otras temáticas, lo cual se relaciona con la deficiente educación sexual en el país. Un estudio relativo a educación sexual sitúa a Chile en el último lugar a nivel Latinoamericano, debido principalmente a la escasa implementación de temáticas relacionadas con educación sexual(27). Para generar cambios con respecto al uso de protección para esta práctica, deben ocurrir cambios sustanciales tanto en actitudes como en normas sociales. Los hallazgos sugieren que al estudiar la práctica de sexo oral se necesita ser conscientes de la interacción con variables sociales, de actitud y de su relación con otras prácticas sexuales.

Una de las limitaciones del estudio se relaciona con el número de hombres participantes $(n=12)$, no pudiendo establecerse diferencias significativas en cuanto a género. Su menor representación evidencia normas sociales que regulan el comportamiento sexual, en donde se le atribuye a la mujer mayor consciencia, responsabilidad y prudencia en el ejercicio de su sexualidad, mientras que a los hombres se les permite excusarse de responsabilidades u omitir aspectos antes mencionados. Por tal razón se podría esperar que los hombres se interesen menos por esta temática que las mujeres. Por otro lado, es necesario reflexionar el por qué Chile se posiciona con el mayor aumento de casos de VIH en Latinoamérica en el período 2010-2017 y cuál es el grado de responsabilidad de las instituciones educacionales a nive país ${ }^{(28)}$.

En este estudio se han develado nuevas evidencias para el abordaje preventivo en las prácticas sexuales desde la odontología, abriendo paso a estudios posteriores para ampliar el alcance de sus conclusiones, orientando una adecuada intervención y prevención hacia la comunidad.

\section{CONCLUSIONES}

- Los conocimientos, conductas y motivos en salud sexual de los/las participantes denotan un conocimiento deficiente en relación a los métodos preventivos para prácticas de sexo oral y al riesgo asociado a esta práctica.

- Los/las estudiantes presentaron deficiencias para reconocer correctamente las formas no efectivas de transmisión del VIH y VPH, denotando una falta de conocimientos respecto a la forma de contagio e indicando la existencia de mitos en la población joven respecto a las vías de transmisión de estos.

- Se visibiliza la necesidad de información con respecto a la forma de realización del test de VIH en el sistema de salud.

- Se evidenció una menor tasa del uso de una barrera de protección para las prácticas de sexo oral en comparación a las otras prácticas estudiadas.

\section{RELEVANCIA CLÍNICA}

Este estudio aporta, por un lado, información relevante respecto de una temática novedosa y necesaria, y por otra, nuevas evidencias para e abordaje preventivo en relación a prácticas sexuales desde lo odontológico, abriendo paso a estudios posteriores para ampliar el alcance de sus conclusiones. La relevancia práctica del estudio radica principalmente en la necesidad de implementar la temática en el currículo de odontología, para fomentar la educación y la investigación en el área, así como orientar una adecuada intervención y prevención con la comunidad y en las propias prácticas de los estudiantes.

\section{FUENTE DE FINANCIAMIENTO} $18 / 002$

Facultad de Odontología de la Universidad de Chile. Proyecto PRI ODO

\section{CONFLICTO DE INTERÉS}

Los autores declaran no tener ningún conflicto de interés.

\section{Bibliografía}

1. Corona F, Funes F. Abordaje de la sexualidad en la adolescencia. Rev Med Clin Condes. 2015; 26(1): 74-80.

2. Palma I (2006). Sociedad chilena en cambio, sexualidades en transformación. (Tesis doctoral). Santiago, Universidad de Chile, 2006.

3. OMS. Estrategia mundial del sector salud contra las infecciones de transmisión sexual 2016-2021. Hacia el fin de las ITS. 2016.

4. Moreno C. La sexualidad en contextos de transformación. Material del curso "Sexualidades en la escuela" impartido en U Abierta, Universidad de Chile; 2015.

5. González E, Molina T, Luttges C. Características de la educación sexual escola recibida y su asociación con la edad de inicio sexual y uso de anticonceptivos en adolescentes chilenas sexualmente activas. Rev Chil Obstet ginecol. 2015; 80: 24-32 6. Urtubey S. Erotismo femenino: la llave de la sexualidad contemporánea. Ed. Círculo latino; 2005.

7. Saini R, Saini S, Sharma S. Oral sex, oral health and orogenital infections. J Global Infect Dis. 2010; 2: 57-62.

8. Conard $L$ y Blythe $M$. Sexual function, sexual abuse and sexually transmitted diseases in adolescence. Best Practice \& Research: Clinical Obstetrics \& Gynaecology. 2003; 17: 103-16.

9. Kunar T, Puri G, Konidena A, Arora N, Patil D, Gupta R. Oral sex and oral Health An enigma in itself. Indian J Sex Transm Dis AIDS. 2015; 36(2): 129-132.

10. Durán A, Rodríguez M, De la Teja E, Zebadúa M. Succión, deglución, masticación y sentido del gusto prenatales. Desarrollo sensorial temprano de la boca. Acta Ped Mex. 2012; 33: 137-141.

11. Chiego D. Principios de histología y embriología bucal con orientación clínica. 4ta ed. Barcelona: Elsevier España, S.L; 2014

12. Gautier R y Boeree G. Teorías de la Personalidad: una selección de los mejores autores del S. XX. Ed. UNIBE; 2005.

13. Cortés N. La succión: una actividad erótica. Revista Facultad de Odontología Universidad Antioquía. 2010; 11: 47-50.

14. Espada J y Orgilés $M$. Riesgo sexual en adolescentes según la edad de debut sexual. Acta Colombiana de Psicología. 2014; 17: 53-60.

15. OPS. Recomendaciones básicas: Profilaxis post exposición ocupacional y no ocupacional al VIH, Virus de la hepatitis B y C y normas de bioseguridad. Cuarta edición, 2013.
16. MINSAL. Normas de profilaxis, diagnóstico y tratamiento de las infecciones de transmisión sexual. Ministerio de Salud; 2016.

17. Blanc A, Rojas A. Uso del preservative, número de parejas y debut sexual en jóvenes en coito vaginal, sexo oral y sexo anal. Rev Int Androl. 2018; 16(1): 8-14

18. Rodríguez C, Colazo E, Dodge B, Roman A. "Sexplorando": sexual practices and condom use among an internet- based sample of men and women in Puerto Rico. J Sex Med. 2014; 11: 2385-2395

19. Schuster M, Bell R, Kanouse D. The sexual practices of adolescent virgins: Genital sexual activities of high school students who have never had vaginal intercourse. AJPH. 1996; 86: 1570-6.

20. Schwartz I. Sexual activity prior to coital initiation: A comparison between males and females. Arch Sex Behav. 1999; 28: 63-9.

21. Halpern-Felsher BL, Cornell JL, Kropp RY, Tschann JM. Oral versus vaginal sex among adolescents: Perceptions, attitudes, and behavior. Pediatrics. 2005; 115 845-51.

22. Valle G, Hernández S. Oral sex and condom use in a US National sample of adolescents and young adults $\mathrm{J}$ of Adols Health. 2018; 62(4): 40-410.

23. Saini R, Saini S, Sharma S (2010). Oral sex, oral health and orogenital infections. J Glob Infect Dis 2: 57-62. 1)

24. Díaz S, Arrieta K, De la Parra E, Muñoz L, Ordoñez M. Conocimientos, actitudes y prácticas relacionadas con la salud sexual y reproductiva en estudiantes de odontología de la Universidad de Cartagena. Universidad de Cartagena. Facultad de Odontología. Departamento de Investigación; 2013.

25. Santana N, Santos T, Sato A, Peder L, Boer C, Sela V, Consolaro M, Teixeira J. Vertical transmission of human papilloma virus in pregnancy: a systematic review and meta-analysis. Int J of Infecc Dis. 2018; 73: 334- 335.

26. OMS. Estrategia mundial del sector de la salud contra las infecciones de transmisión sexual 2016-2021, hacia el final de las ITS. 2016

27. Hunt F, Monterrosas E, Mimbela R. Evaluación de la implementación de la declaracion ministerial "Prevenir en Educación", su cumplimiento en América Latina 2008 - 2015; México, 2015.

28. Global Aids Update. Ending AIDS: Progress towards the 90-90-90 targets. 2017; 130-139. 